\title{
Selective Innervation of Neostriatal Interneurons by a Subclass of Neuron in the Globus Pallidus of the Rat
}

\author{
Mark D. Bevan,, ${ }^{1,2}$ Philip A. C. Booth, ${ }^{1}$ Sean A. Eaton, ${ }^{1}$ and J. Paul Bolam ${ }^{1}$ \\ ${ }^{1}$ Medical Research Council Anatomical Neuropharmacology Unit and 2University Department of Pharmacology, \\ Oxford, OX1 3TH, United Kingdom
}

\begin{abstract}
A subpopulation of neurons in the globus pallidus projects to the neostriatum, which is the major recipient of afferent information to the basal ganglia. Given the moderate nature of this projection, we hypothesized that the pallidostriatal projection might exert indirect but powerful control over principal neuron activity by targeting interneurons, which comprise only a small percentage of neostriatal neurons. This was tested by the juxtacellular labeling and recording of pallidal neurons in combination with immunolabeling of postsynaptic neurons.

In addition to innervating the subthalamic nucleus and output nuclei, 6 of 23 labeled pallidal neurons projected to the neostriatum. Both the firing characteristics and the extent of the axonal arborization in the neostriatum were variable. However, light and electron microscopic analysis of five pallidostriatal neurons revealed that each neuron selectively innervated neostriatal interneurons. A large proportion of the boutons of an individual axon (19-66\%) made contact with parvalbumin-
\end{abstract}

The globus pallidus (GP) or the external segment of the globus pallidus of primates (GPe) is located in a central and theoretically executive position in the basal ganglia macrocircuit and is known to perform several critical operations that influence the output of this system in health and disease (for review, see Albin et al., 1989; DeLong, 1990; Gerfen and Wilson 1996; Smith et al., 1998). It has been suggested that neurons of the GP/GPe are components of the indirect pathway/network that increases the activity of basal ganglia output neurons in a complex spatial and temporal pattern during cortical arousal associated with movement, effects that are hypothesized to underlie the prevention, selection, and/or termination of movement (DeLong, 1971; DeLong et al., 1986; Chevalier and Deniau, 1990; Anderson and Turner, 1991; Ryan and Clark, 1991, 1992; Fujimoto and Kita, 1992, 1993; Ryan and Sanders, 1993, 1994; Turner and Anderson, 1997). In Parkinson's disease, abnormal underactivity of GP/GPe may contribute to excessive inhibition of basal ganglia targets leading to akinesia (for review, see Albin et al., 1989; Crossman 1989; DeLong, 1990), whereas abnormal, rhythmic, synchronized burst-

Received May 5, 1998; revised Aug. 26, 1998; accepted Sept. 3, 1998.

This work was supported by the Wellcome Trust and the Medical Research Council UK. M.D.B. is an Advanced Training Fellow of the Wellcome Trust $(046613 / Z / 96 / Z)$. We gratefully acknowledge Dr. Y. Kaneoke for providing and modifying the program for analysis of bursting in spike trains; Dr. P. Emson for the gift of the nitric oxide synthase antibody; Drs. C. J. Wilson, B. D. Bennett, and E. Stern for helpf ul comments on the analysis and interpretation of this work; and Liz Norman, Caroline Francis, and Paul Jays for technical assistance.

Correspondence should be addressed to Dr. Mark D. Bevan, Medical Research Council Anatomical Neuropharmacology Unit and University Department of Pharmacology, Mansfield Road, Oxford, OX1 3TH, UK.

Copyright (C) 1998 Society for Neuroscience $\quad 0270-6474 / 98 / 189438-15 \$ 05.00 / 0$ immunoreactive interneurons. An individual parvalbuminimmunoreactive neuron ( $n=27$ ) was apposed on average by 6.7 boutons $(\mathrm{SD}=6.1)$ from a single pallidal axon $(n=2)$. Individual pallidostriatal boutons typically possessed more than one symmetrical synaptic specialization. In addition, 3-32\% of boutons of axons from four of five pallidal neurons contacted nitric oxide synthase-immunoreactive neurons. Descending collaterals of pallidostriatal neurons were also found to make synaptic contact with dopaminergic and GABAergic neurons of the substantia nigra. These data imply that during periods of cortical activation, individual pallidal neurons may influence the activity of GABAergic interneurons of the neostriatum (which are involved in feed-forward inhibition and synchronization of principle neuron activity) while simultaneously patterning neuronal activity in basal ganglia downstream of the neostriatum.

Key words: globus pallidus; neostriatum; interneuron; cortex; feed-forward inhibition; synchronization

ing of GP/GPe neurons may help to entrain the low-frequency oscillatory output of the basal ganglia during resting tremor (Nini et al., 1995; Chockkan et al., 1997). Recent studies suggest that the GP/GPe and their limbic homolog the ventral pallidum also subserve an important integrative role through their divergent projections to the subthalamic nucleus (STN) and output nuclei, which distribute information between the functionally diverse parallel pathways that course through the basal ganglia (Bevan et al., 1996, 1997a).

An emergent property of GP/GPe neurons that has yet to be incorporated into models of basal ganglia function is the widespread targets of their axons (Smith and Bolam, 1991; Bolam and Smith, 1992; Bevan et al., 1994a,b; Kita and Kitai, 1994; Smith et al., 1994; Shink et al., 1996; Nambu and Llinas, 1997; Sato et al., 1997). Thus a single neuron may project to all nuclei of the basal ganglia and through their large, multiple, and proximally placed GABAergic axon terminals bind or orchestrate coherent network activity (Smith and Bolam, 1991; Bolam and Smith, 1992; Bevan et al., 1994a,b, 1996, 1997a; Smith et al., 1994; Shink et al., 1996). An analogous operation has been described for GABAergic interneurons of the cortex, which share many similarities with GP/GPe neurons (Cobb et al., 1995; Whittington et al., 1995).

A subpopulation of GP neurons projects to the neostriatum (Staines et al., 1981; Beckstead, 1983; Jayaraman, 1983; Staines and Fibiger, 1984; Smith and Parent, 1986; Shu and Peterson, 1988; Walker et al., 1989; Kita and Kitai, 1994; Rajakumar et al., 1994; Spooren et al., 1996; Nambu and Llinas, 1997), which is the major input structure of the basal ganglia and the major source of afferents to the GP/GPe (for review, see Gerfen and Wilson, 
1996; Smith et al., 1998). The findings of preliminary studies (Staines and Hincke, 1991; Bennett et al., 1993) imply that the pallidostriatal projection might exert widespread effects on the processing and transmission of information by the neostriatum by selective innervation of neostriatal interneurons that represent only a small fraction of neurons in this nucleus. Therefore, the first objective of the study was to determine directly the extent and the synaptic targets of the pallidostriatal projection.

Two major firing patterns are observed in GP/GPe neurons in vivo (DeLong, 1971; Filion, 1979; Kita and Kitai, 1991; Ryan and Sanders, 1993), and intracellular recording studies indicate that two major types of GP neuron can be differentiated on the basis of their membrane and firing properties (Kita and Kitai, 1991; Nambu and Llinas, 1994, 1997). The second objective therefore was to determine the firing patterns of neurons that project to the neostriatum. To examine these issues we combined physiological recording and labeling of single GP neurons in vivo (Pinault, 1996) in combination with multiple immunocytochemical staining of neostriatal interneurons.

Some of this work has been published previously in abstract form (Bevan et al., 1997b).

\section{MATERIALS AND METHODS}

\section{Recording and filling of GP neurons}

Procedures involving animals were performed in strict accordance with the Animals (Scientific Procedures) Act, 1986 (UK), and with the Society for Neuroscience policy on the use of animals in neuroscience research. Extracellular recordings of neurons in the GP of male Wistar rats (200-350 gm; Charles River, Margate, Kent, UK) that were deeply anesthetized with ketamine $(90 \mathrm{mg} / \mathrm{kg}$, i.p.) and xylazine $(10 \mathrm{mg} / \mathrm{kg}$, i.p.) were made under stereotaxic guidance (David Kopf Instruments, Tujunga, CA; Inchworm, Burleigh Instruments, New York, NY) using 15-20 M $\Omega$ glass pipettes containing $1.5 \%$ neurobiotin (Vector, Peterborough, UK) in $0.5 \mathrm{M} \mathrm{NaCl}$. The signal was amplified $(10 \times)$ using an Axoclamp 2A (Axon Instruments, Foster City, CA) and an AC/DC amplifier (100×; NL 106, Digitimer, Welwyn Garden City, UK), filtered (0.3-5 kHz; NL125, Digitimer) and viewed on a digital oscilloscope (Gould 4164, Gould Instruments, Ilford, Essex, UK). Single units that were often several millivolts in amplitude were easily discriminated from noise, and extracellularly recorded action potentials were used to trigger a digital pulse (NL 201 Spike Trigger, Digitimer) to a laboratory interface (CED 1401Plus, Cambridge Electronic Design, Cambridge, UK) and then to a computer running the Spike 2 data acquisition and analysis program (Cambridge Electronic Design). Recordings of spontaneous activity of 90-120 sec duration were made. Injections of 1-10 nA cathodal current at a $200 \mathrm{msec}$ on/off cycle were then made as described previously (Pinault, 1996), using the bridge circuitry of the intracellular amplifier as the pipette was slowly advanced onto the neuron. When the firing pattern of the neuron was robustly modulated on the positive phase of the duty cycle, the advance of the electrode was terminated, and the magnitude of injected current was reduced to prevent damage. Neurons were typically modulated for at least $20 \mathrm{~min}$ to obtain reliable filling. After the injection $(10-14 \mathrm{hr})$, the animal was given a lethal dose of pentobarbitone and perfused via the ascending aorta with $100 \mathrm{ml}$ of saline followed by $300 \mathrm{ml}$ of $0.3 \%$ glutaraldehyde and $3 \%$ paraformaldehyde in $0.1 \mathrm{M}$ phosphate buffer, $\mathrm{pH} 7.4$, and then by $200 \mathrm{ml}$ of the same solution without glutaraldehyde.

\section{Visualization of filled cells and their postsynaptic targets}

Standard histochemical and immunocytochemical procedures previously reported for the visualization of filled cells and their targets were used in this study, and so these will be described only briefly. For comprehensive details of these procedures the reader is referred to previous publications (Bolam, 1992; Bevan et al., 1997a). Vibratome sections $(50-60 \mu \mathrm{m})$ of the fixed brain were made in the sagittal plane. Sections were made permeable using $0.3 \%$ Triton X-100 (Sigma, Dorset, UK) in incubation solutions for light microscopy and by multiple freeze-thawing cycles in cryoprotectant, cooled isopentane and liquid nitrogen for electron microscopy. Neurobiotin in the recorded cell was revealed by incubation in avidin-biotin peroxidase complex (ABC, 1:100, Vector) for $24-36 \mathrm{hr}$ at $4^{\circ} \mathrm{C}$ and then in hydrogen peroxide and diaminobenzidine tetrahydrochloride (DAB; Sigma) in the absence or presence of nickel ions (NiDAB). Parvalbumin-immunoreactive (PV-IR) structures were revealed by incubation in mouse anti-PV (1:2000; SWant, Bellinzona, Switzerland) for $24-36 \mathrm{hr}$ at $4^{\circ} \mathrm{C}$, donkey anti-mouse $\operatorname{IgG}$ (1:100; Jackson ImmunoResearch, West Grove, PA) for $2 \mathrm{hr}$ at room temperature, and mouse peroxidase anti-peroxidase (PAP; 1:100; Dako, Glostrup, Denmark) for $2 \mathrm{hr}$ at room temperature, and the chromogen that was not used to reveal the recorded cell, i.e., DAB or Ni-DAB. Sections were then divided along the GP/neostriatum border using a razor blade under a dissecting microscope. Nitric oxide synthase-immunoreactive (NOSIR) structures in the neostriatum were revealed by incubation in sheep anti-NOS (1:1000; gift from Dr. Piers Emson) (Herbison et al., 1996) for 24-36 hr at $4^{\circ} \mathrm{C}$, donkey anti-sheep IgG (1:100; Jackson ImmunoResearch) for $2 \mathrm{hr}$ at room temperature, and sheep PAP (1:100; Jackson ImmunoResearch) for $2 \mathrm{hr}$ at room temperature. Peroxidase bound to NOS-IR structures was detected using Vector VIP (light microscopy) or benzidine dihydrochloride (BDHC; electron microscopy) (Bolam, 1992). Tyrosine hydroxylase-immunoreactive (TH-IR) structures caudal to the neostriatum were revealed by incubation in rabbit anti-TH $(1: 1000$; Eugenetech International) for $24-36 \mathrm{hr}$ at $4^{\circ} \mathrm{C}$, donkey anti-rabbit IgG (1:100; Jackson ImmunoResearch) for $2 \mathrm{hr}$ at room temperature, and rabbit PAP (1:100; Dako) for $2 \mathrm{hr}$ at room temperature. Peroxidase bound to TH-IR structures was detected as for nitric oxide synthase. Sections were mounted for light microscopy or electron microscopy as described previously (Bolam, 1992).

\section{Electrophysiological analysis}

Spike times obtained from Spike 2 were converted into interspike intervals using Excel (Microsoft, Redmond, WA). The coefficient of variation of interspike interval for each cell was calculated from the SD of the last 499 interspike intervals in the recording divided by their average interspike interval (Johnson, 1996). Firing frequency of the same spike train was calculated from the reciprocal of the mean interspike interval. The burst index of the same spike train was calculated using an algorithm based on discharge density, as described previously (Kaneoke and Vitek, 1996).

\section{Anatomical analysis}

Light microscopy. Juxtacellularly labeled GP neurons and randomly selected postsynaptic PV-IR neurons were reconstructed in two dimensions under 50-100× oil immersion objectives using a drawing tube attached to a light microscope (Dialux 22, Leica, Milton Keynes, UK). In the neostriatum, the position and structure to which each axonal bouton was apposed were recorded. The target was recorded as unknown when immunoreagents had not penetrated to the depth at which the bouton was located and recorded as negative when the bouton was not apposed to an immunoreactive structure but was located at a depth to which immunoreagents had penetrated.

Electron microscopy. In some cases, GP boutons that were identified by light microscopy as being apposed to PV-IR and NOS-IR neurons in the neostriatum and TH-IR and PV-IR neurons of the substantia nigra were resectioned on an ultramicrotome (Reichert Ultracut E, Leica) and further analyzed by electron microscopy (Phillips CM10, Eindhoven, The Netherlands) as described previously (Bolam, 1992; Bevan et al., 1997a).

\section{RESULTS}

On the basis of their axonal projections, two types of neuron were labeled by the juxtacellular application of neurobiotin in the GP. One population (6 of 23 ) projected to the neostriatum in addition to locally within the GP and caudally within the basal ganglia; these neurons are the focus of this report (Fig. 1). The second population (17 of 23) projected within the GP and caudally within the basal ganglia (Fig. 2) and are the subject of a future report.

\section{Firing patterns of pallidostriatal neurons}

A spectrum of firing frequencies and patterns was found in pallidal neurons that project to the neostriatum (Table 1). Neurons firing at relatively low and higher frequencies fired regularly, irregularly, or in bursts (Fig. 3A,B; Table 1). Pallidal neurons were completely labeled with tracer when firing was consistently 


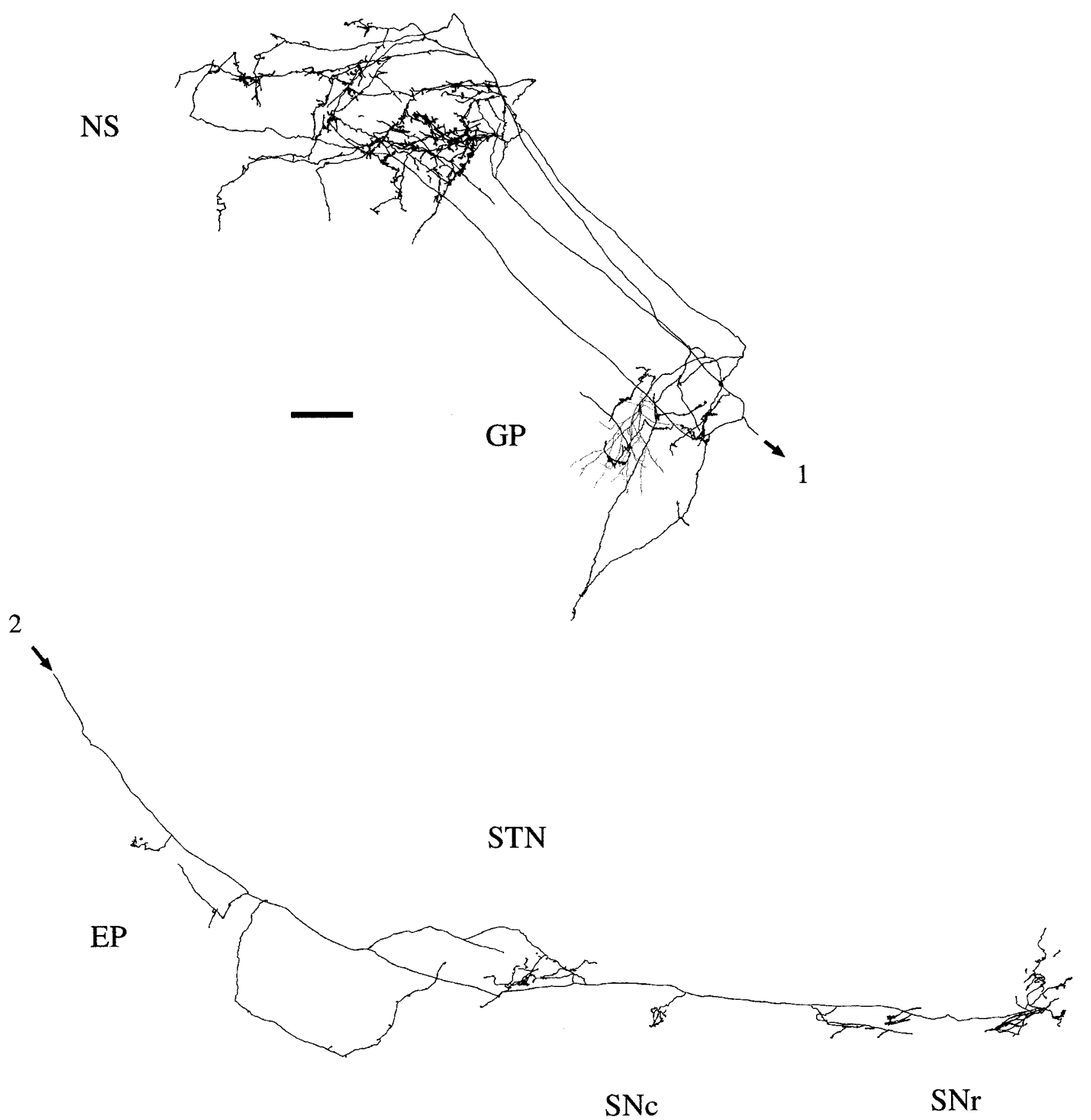

Figure 1. Reconstruction along the rostrocaudal axis of the axon (black; 1 connects to 2 ) and soma and dendrites ( gray) of a single GP neuron [neuron 9678 (Table 1)] that projects to the neostriatum $(N S)$ in addition to other basal ganglia nuclei. Note the extensive axonal arborization in the neostriatum (1353 boutons) that arises from four branches of the main axon. Boutons were located in 27 sagittal sections of $50 \mu \mathrm{m}$ thickness. Note also the local collaterals in the GP (315 boutons) that extend well beyond the dendrites of the parent neuron. This cell also innervated the entopeduncular nucleus $(E P)$ (27 boutons), the subthalamic nucleus (STN) (66 boutons), and the substantia nigra ( 247 boutons). In the substantia nigra the axon gave rise to three arborizations at three rostrocaudal levels. The most rostral arborization was restricted to the rostral part of substantia nigra pars compacta (SNc) (29 boutons). The two more caudal arborizations were located in the substantia nigra pars reticulata ( $S N r)(87$ and 131 boutons). Scale bar, $300 \mu \mathrm{m}$.

and robustly modulated by the juxtacellular application of positive current for at least 20 min (Fig. 3C; see Fig. $5 A, F$ ), which was followed by the recovery of normal firing pattern.

\section{Appearance of staining}

\section{Light microscopy}

Juxtacellularly labeled neurons were filled with a dense blueblack reaction product when visualized with Ni-DAB (see Figs.
$5 A-E, 6 A, 7 A, 8 B, C, 9 B-D)$ and a dense brown reaction product when visualized with DAB (see Figs. $5 F, G$ ). Postsynaptic structures were labeled with a dense brown (see Figs. 5B,D,E, 6A, $9 B-D$ ), blue-black (see Fig. $5 F$ ), or purple (see Fig. $5 C, D, G$ ) reaction product when visualized with $\mathrm{DAB}$, NiDAB, or VIP, respectively. Postsynaptic structures labeled with BDHC contained a dense blue crystalline reaction product (see Figs. $7 A, 8 B, C)$. 


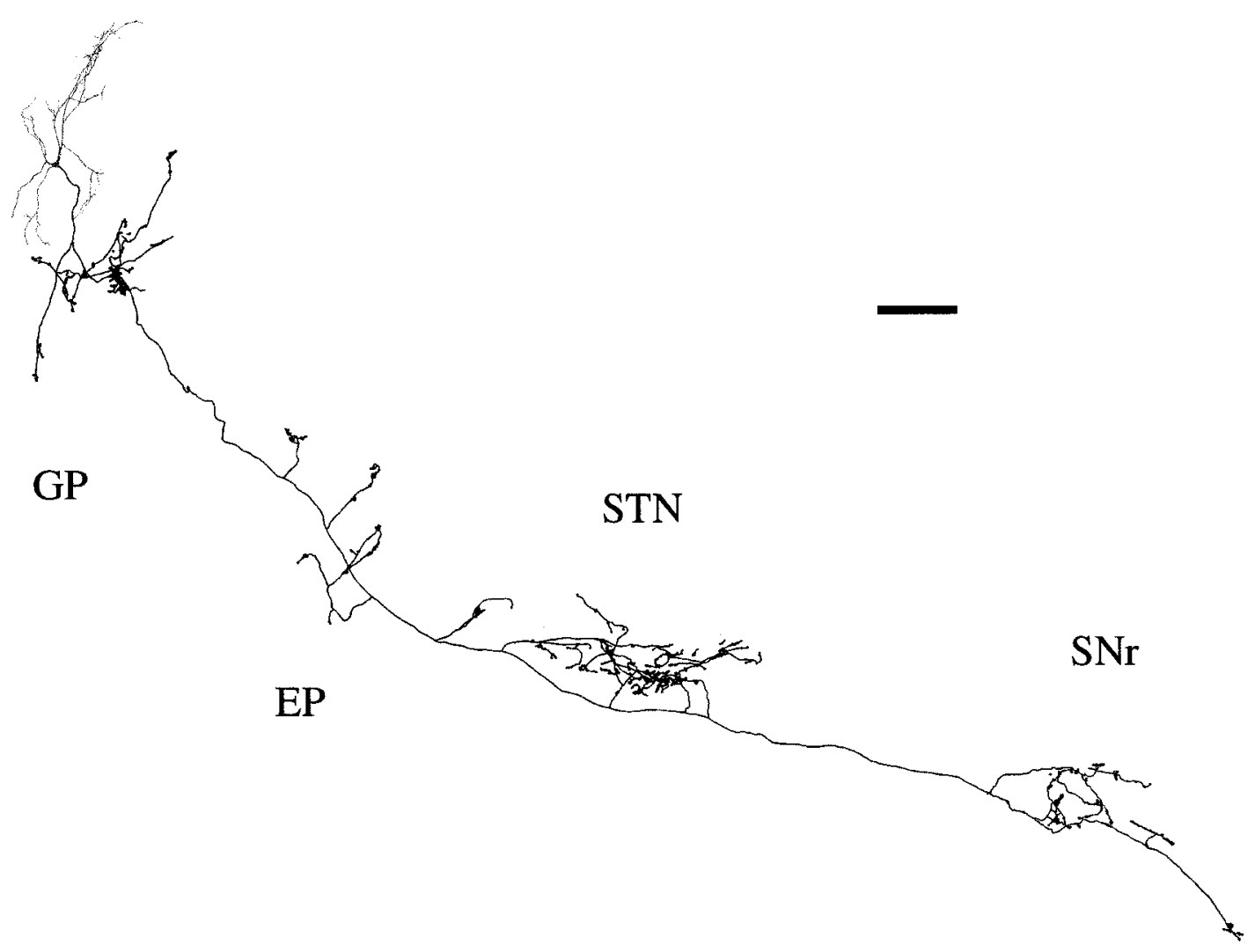

Figure 2. Reconstruction along the rostrocaudal axis of the axon (black) and the soma and dendrites ( gray) of a representative example of the majority of GP neurons, i.e., those that do not project to the neostriatum. These neurons give rise to local axonal arborizations (145 boutons in this example) and project to the entopeduncular nucleus $(E P)$ (43 boutons in this example), the subthalamic nucleus $(S T N)$ (181 boutons in this example), and the substantia nigra $(S N)$ (120 boutons in this example). Scale bar, $300 \mu \mathrm{m}$.

Table 1. Physiological properties of the six pallidal neurons that projected to the neostriatum

\begin{tabular}{lcll}
$\begin{array}{l}\text { Neuron } \\
\text { number }\end{array}$ & $\begin{array}{l}\text { Mean firing } \\
\text { rate }(\mathrm{Hz})\end{array}$ & C.V. & Burst index \\
\hline 9645 & 17.56 & 1.45 & 0.33 \\
9665 & 9.60 & 1.70 & 2.00 \\
9666 & 16.10 & 0.76 & 0.00 \\
9671 & 6.28 & 1.27 & 0.00 \\
9672 & 17.92 & 0.94 & 0.00 \\
9678 & 12.48 & 0.70 & 1.00 \\
\hline
\end{tabular}

C.V., Coefficient of variation of the interspike interval.

\section{Electron microscopy}

Juxtacellularly labeled neurons that were visualized with Ni-DAB were filled with an amorphous electron-dense reaction product that adhered to or invaded subcellular organelles (see Figs. $6 B-H, 7 B-H, 8 A, D-I, 9 A, E-H)$. Parvalbumin immunoreactivity was visualized with $\mathrm{DAB}$, which was similar in appearance to Ni-DAB although generally much less dense (see Figs. 6B-D). Presynaptic and postsynaptic elements labeled with DAB-based reaction products could only be distinguished unequivocally in the electron microscope by correlated light and electron microscopy (see Figs. 6, 9).

Nitric oxide synthase-immunoreactive and TH-IR structures were visualized with BDHC (see Figs. $6 A, H, 7 B-H, 8 A, D-I$ ), which formed a more electron-dense and crystalline reaction product than DAB-based products and did not have a particular association with subcellular organelles.

\section{Location and somatodendritic features of pallidostriatal neurons}

The six labeled pallidostriatal neurons in this study were located in the dorsal and medial two-thirds of the rostrocaudal extent of the GP (Table 2). The dimensions and branching patterns of their dendrites are consistent with earlier reports (see Discussion). Thus each neuron gave rise to three to five primary dendrites that branched repeatedly and extended over a large fraction of the nucleus. Each neuron bore dendritic spines but the size, distribution, and density were variable between neurons and even between the dendrites of the same neuron. Complex varicose dendritic endings were noted on some of the tertiary dendrites of all neurons. In two neurons, one of the dendrites extending in the dorsal direction appeared to be damaged by the injection procedure; they were shrunken and exhibited signs of degeneration. We cannot comment on the three-dimensional shape of the dendritic arborization because a two-dimensional tracing technique was used.

\section{General features of axonal arborizations of pallidostriatal neurons}

In addition to projecting to the neostriatum, all six neurons gave rise to local collaterals within the GP (Figs. 1, 4). Their relationship to the parent dendritic tree and the number and density of varicosities on these local collaterals were variable. Of five neurons whose axons were traced caudally out of the GP (neurons 


\section{A + || | || ||||| |||||| ||| ||1||||||||||||||||||| |||}

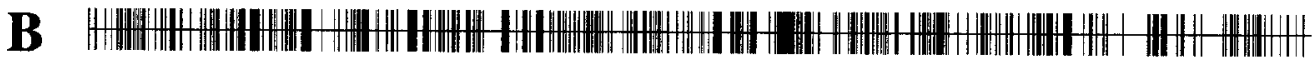

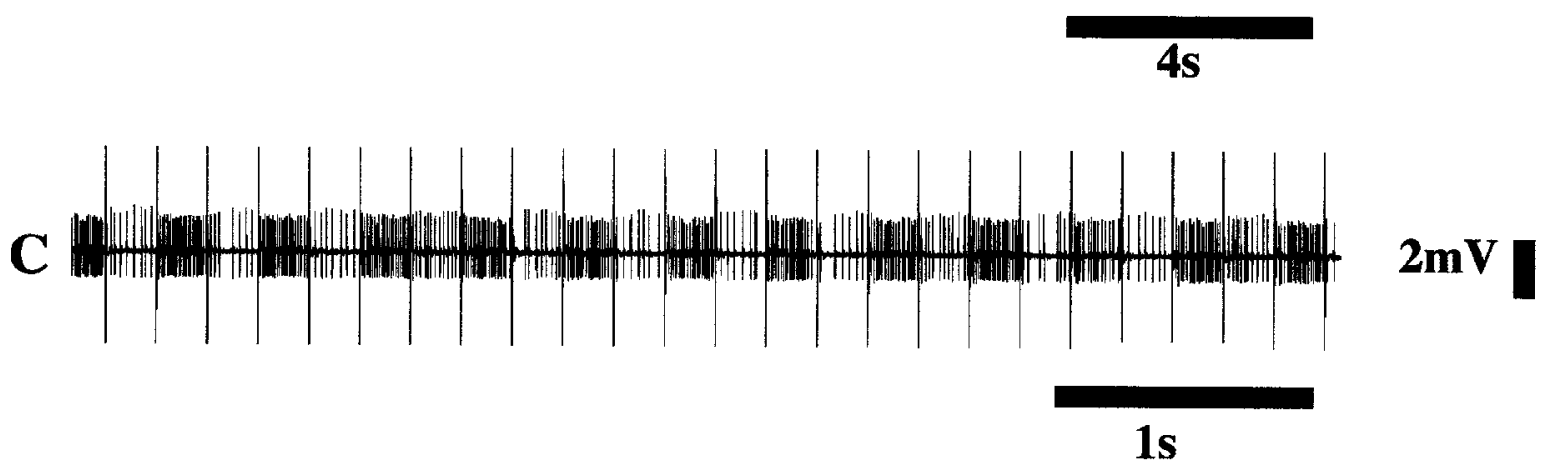

Figure 3. Physiological recordings from the GP of anesthetized rats. $A$, Triggered spike train of neuron 9665 (Table 1). This neuron possessed a relatively low mean firing rate and discharged in a bursting pattern. $B$, Triggered spike train of neuron 9672 (Table 1). This neuron discharged at a relatively high mean rate in an irregular, nonbursting pattern. $C$, Representative example of the modulation of spontaneous firing of a neuron in the GP during a successful juxtacellular injection. Note the alternate $200 \mathrm{msec}$ periods of higher-frequency firing during current injection and lower-frequency firing when current injection was ceased. The onset and termination of current injection can be noted from the large switching artifact, which is of greater amplitude than the extracellularly recorded waveform.

Table 2. Positions within GP of the six neostriatal projecting neurons labeled in this study

\begin{tabular}{|c|c|c|c|c|c|c|c|c|c|}
\hline \multirow{3}{*}{$\begin{array}{l}\text { Neuron } \\
\text { number }\end{array}$} & \multicolumn{9}{|c|}{ Axis/region of GP } \\
\hline & \multicolumn{3}{|c|}{ Dorsoventral } & \multicolumn{3}{|c|}{ Rostrocaudal } & \multicolumn{3}{|c|}{ Mediolateral } \\
\hline & Dorsal & Middle & Ventral & Rostral & Middle & Caudal & Medial & Middle & Lateral \\
\hline 9645 & & + & & & + & & + & & \\
\hline 9665 & + & & & & & + & + & & \\
\hline 9666 & + & & & & + & & & + & \\
\hline 9671 & & + & & + & & & + & & \\
\hline 9672 & + & & & & + & & + & & \\
\hline 9678 & + & & & + & & & & + & \\
\hline
\end{tabular}

The positions are defined according to dorsoventral, rostrocaudal, and mediolateral axes that are divided into thirds.

9645, 9665, 9671, 9672, and 9678), all gave rise to axonal arborizations in the subthalamic nucleus and the substantia nigra, and four of five also terminated in the entopeduncular nucleus. Similarly, the dimensions and number and density of varicosities on these collaterals were highly variable.

\section{Neostriatal axonal arborizations}

Neostriatal arborizations arose from one to five branches of the main axon that coursed laterally before proliferating medially (Figs. 1, 4). The general course of these axons was along the axis defined by the corticofugal and striatofugal fiber bundles that converged onto the labeled cell. The dimensions of the neostriatal axonal arborizations were variable. In one case the arborization stretched over several millimeters of the dorsoventral, mediolateral, and rostrocaudal axes of the neostriatum (Fig. 1), whereas in another case a more restricted focal arborization arose from a single branch of the main axon and occupied only a few hundred micrometers of the neostriatum (Fig. 4B). This heterogeneity was reflected in the total numbers of boutons carried by each arborization (Table 3). It is unlikely that this heterogeneity arose from incomplete filling, because the axons of these cells in the neostriatum and substantia nigra did not display attenuation of staining intensity compared with local axons in the GP.

\section{Postsynaptic targets of pallidostriatal neurons in the neostriatum \\ Parvalbumin-immunoreactive neurons}

The neostriatal axons of the five neurons that were examined selectively innervated parvalbumin-immunoreactive neurons (Figs. 5B,D,E, 6A-G; Tables 3, 4) because the proportion of pallidostriatal boutons in contact with parvalbumin-immunoreactive neurons exceeded the proportion that this class of neuron represents in the neostriatum. All parts of these interneurons were innervated (Figs. 5B,D,E, 6A-G; Tables 3, 4). Typically, clusters of pallidostriatal axonal varicosities were apposed to restricted parts of the dendrites and/or the soma of individual postsynaptic neurons (Figs. 5B,D,E, 6A-G). In tissue prepared for electron microscopy, these associations were generally verified as synaptic contacts (Fig. 6). Of the boutons apposing PV-IR neurons that were analyzed by electron microscopy, 16 of 18 were confirmed to be synaptic contacts. In the other two cases, the boutons apposed 


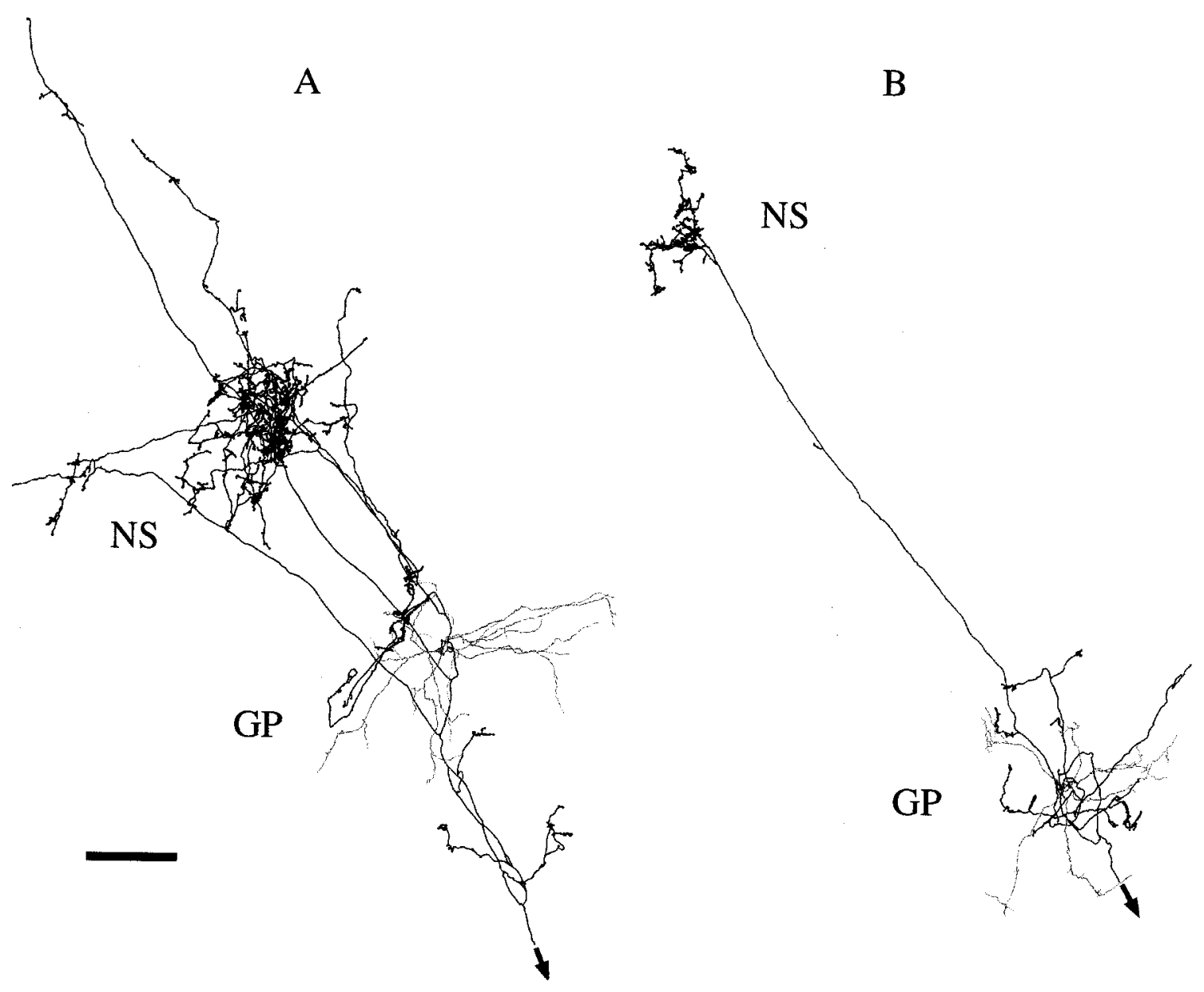

Figure 4. $A, B$, Reconstructions along the rostrocaudal axis of the axon (black) and the soma and dendrites ( gray) of two GP neurons $(A$, neuron 9666 ; $B$, neuron 9672) that project to the neostriatum in addition to other basal ganglia nuclei. Neuron 9666 gave rise to 942 boutons, and neuron 9672 gave rise to 329 boutons in the neostriatum. Note the heterogeneous nature of the local and neostriatal axonal arborizations in terms of their dimensions and density (also see Fig. 1). The arrow indicates the branch of the axon that gave rise to descending projections to the EP, STN, and SNr. Scale bar (shown in $A$ for $A$ and $B$ ): $300 \mu \mathrm{m}$.

Table 3. Numbers of boutons and synaptic targets of the neostriatal collaterals of five completely filled GP neurons

\begin{tabular}{|c|c|c|c|c|c|c|c|}
\hline \multirow{2}{*}{$\begin{array}{l}\text { Neuron } \\
\text { number }\end{array}$} & \multirow{2}{*}{$\begin{array}{l}\text { Number of } \\
\text { boutons }\end{array}$} & \multicolumn{3}{|c|}{ Percentage contacting PV-IR neurons } & \multicolumn{3}{|c|}{ Percentage contacting NOS-IR neurons } \\
\hline & & Soma & Dendrite & Total & Soma & Dendrite & Total \\
\hline 9665 & 478 & 2.4 & 47.7 & 50.1 & 0 & 0 & 0 \\
\hline 9666 & 942 & 8.5 & 57.6 & 66.1 & 2.4 & 6.5 & 8.9 \\
\hline 9671 & 851 & 4.3 & 14.9 & 19.2 & 0.9 & 6.1 & 7.0 \\
\hline 9672 & 329 & 11.6 & 22.5 & 34.1 & 2.4 & 0.6 & 3.0 \\
\hline 9678 & 1353 & 10.2 & 39.0 & 49.2 & 1.4 & 30.9 & 32.3 \\
\hline
\end{tabular}

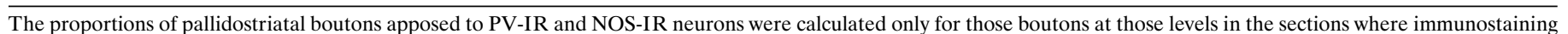

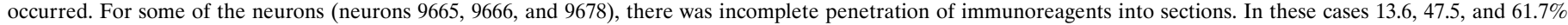
of boutons were at levels devoid of immunostaining, respectively.

the membrane of the putative postsynaptic structure, but the plane of section prevented unequivocal verification of synaptic contact. The terminals formed symmetric synaptic contacts; six were identified in contact with perikarya and 10 with dendrites of PV-IR neurons (Fig. 6). Individual terminals often possessed multiple sites of neurotransmitter release (Fig. 6D,E,G).

Because most of the pallidostriatal boutons in contact with PV-IR neurons formed verifiable synaptic contacts, we made quantitative estimates of the number of pallidostriatal boutons in contact with individual neurons on the basis of light microscopic analysis. Pallidostriatal boutons in contact with randomly selected PV-IR neurons $(n=27)$ in two animals in which the staining of interneurons occurred throughout sections were examined, and the number and position of GP boutons in contact with them were recorded (neurons 9671 and 9672; Table 4). The data derived from the two animals were not signficantly different from one another (Mann-Whitney $U$ test) and therefore were pooled. The pooled data were Poisson in distribution. Parvalbumin- 

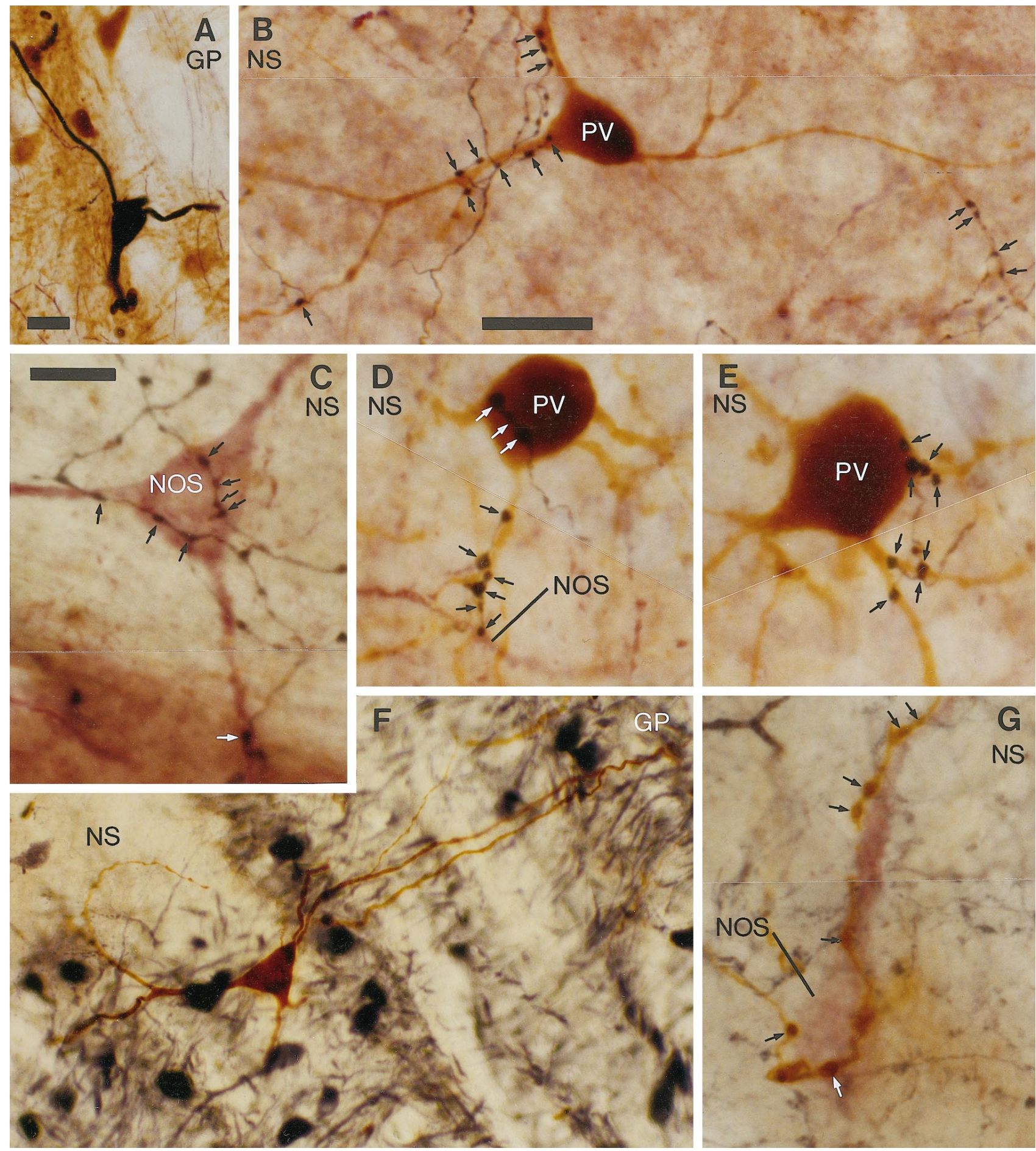

Figure 5. Light micrographs illustrating juxtacellularly labeled GP neurons $(A, F)$ and their axons in the neostriatum (NS) in relation to PV-IR (B, $D$, $E)$ and NOS-IR $(C, G)$ interneurons. In $A-E$, the GP neurons were visualized with Ni-DAB giving a blue-black stain, the PV-IR interneurons were visualized with DAB and stained brown, and the NOS-IR interneurons were visualized with VIP and stained purple. In $F$ and $G$, another combination of chromogens was used: GP neurons were visualized with DAB (brown), PV-IR neurons were visualized with Ni-DAB (blue-black), and NOS-IR interneurons were visualized with Vector VIP ( purple). A, F, Juxtacellularly labeled neurons in the GP in sections that were also stained to reveal PV-IR neurons. Note the Golgi-like labeling of the filled single neurons and the many PV-IR structures. In $F$ the neuron is located on the rostral-dorsal border of the GP, which is clearly defined by high density of PV-IR neurons in the GP compared with the lower density of PV-IR neurons in the neostriatum $(N S) . B, D, E$, Selective innervation of PV-IR interneurons of the neostriatum by GP neurons. Note the clusters of axonal varicosities (arrows) apposing restricted parts of the PV-IR postsynaptic cells. $C, D, G$, Examples of the innervation of NOS-IR interneurons of the NS by the GP. $C, E$, The typical arrangement of multiple pallidal axonal boutons apposing individual postsynaptic neurons (arrows). $D$, A single GP axon forms multiple appositions with a PV-IR neuron (arrows), and in addition apposes a small-diameter dendrite of a NOS-IR interneuron (most ventral arrow). Scale bars (shown in $A$ for $A$ and $F$ ): $20 \mu \mathrm{m} ; B, 20 \mu \mathrm{m}$; (shown in $C$ for $C-E, G$ ): $10 \mu \mathrm{m}$. 

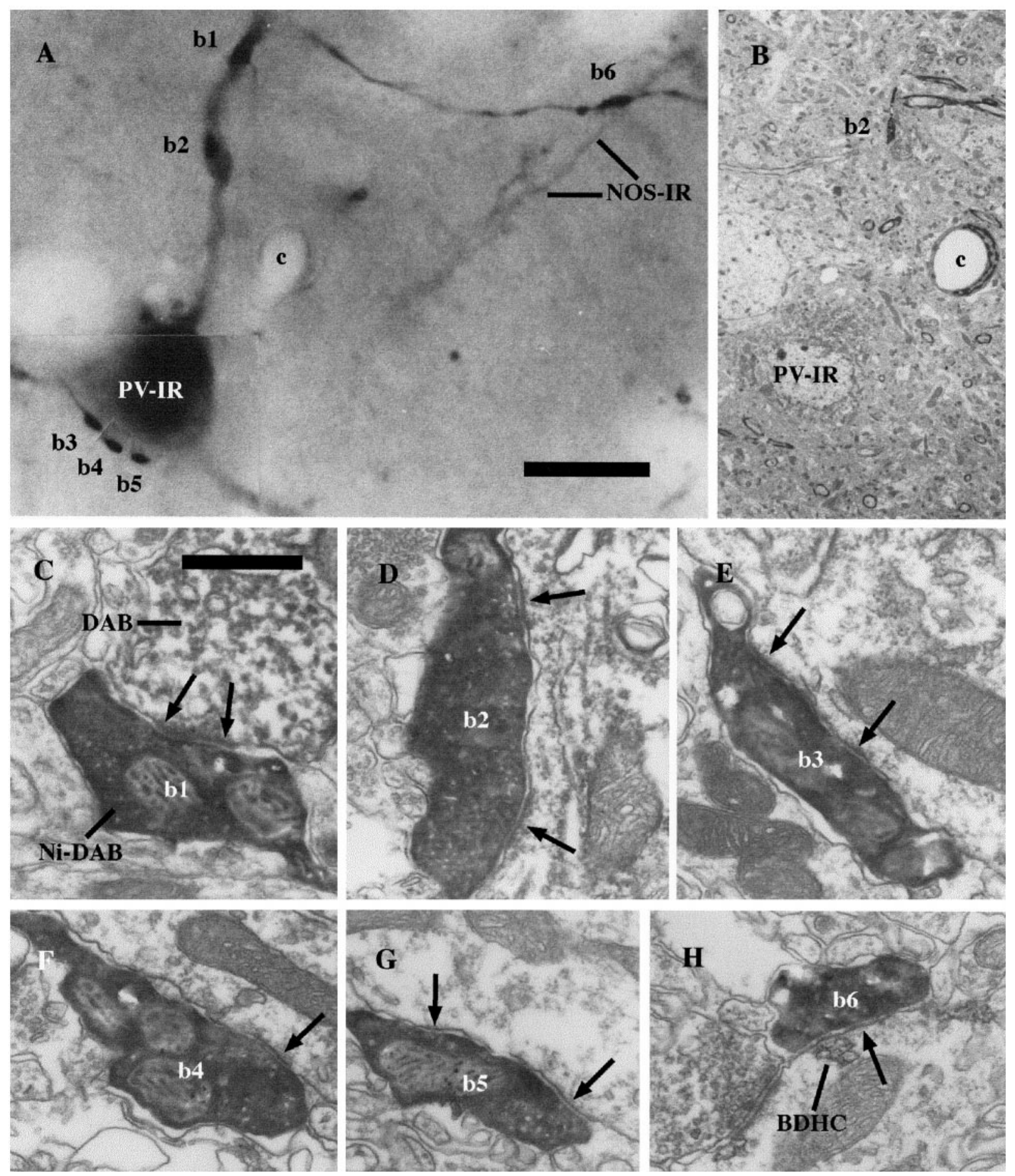

Figure 6. $A-H$, Light and electron micrographs illustrating the selective innervation of neostriatal interneurons by individual neurons of the GP. In these micrographs the axon of the GP neuron 9678 is revealed with Ni-DAB, the PV-IR structures are revealed with DAB, and NOS-immunoreactive structures are revealed with BDHC. $A$, Light microscopic montage of a PV-IR neuron and a NOS-IR dendrite. The PV-IR neuron is apposed by five boutons of the pallidostriatal neuron, two $(b 1, b 2)$ of which contact the proximal dendrite, and three $(b 3-b 5)$ of which contact the perikaryon. The NOS-IR dendrite is also apposed by a bouton $(b 6)$. $B$, Electron micrograph of part of the same region shown in $A$. The soma of the PV-IR neuron, the capillary $(c)$, and the pallidostriatal bouton $(b 2)$ act as registration marks between the two levels of investigation. $C-H$, High-magnification electron micrographs of the pallidostriatal boutons in contact with the PV-IR $(C-G)$ and NOS-IR $(H)$ interneurons. Each bouton forms symmetrical synaptic contact (arrows) with its postsynaptic target. Some boutons possess multiple release sites $(b 2, b 3, b 5)$. At higher magnification the amorphous nature of the NiDAB and DAB reaction products $(C-H)$ and the crystalline nature of the BDHC reaction product $(H)$ are apparent. Scale bars (shown in $A$ for $A$ and $B$ ): $10 \mu \mathrm{m}$; (shown in $C$ for $C, D-H$ ): $0.5 \mu \mathrm{m}$. 


\begin{tabular}{|c|c|c|c|c|}
\hline $\begin{array}{l}\text { PV-IR neuron } \\
\text { number }\end{array}$ & Soma & $\begin{array}{l}\text { Primary } \\
\text { dendrite }\end{array}$ & $\begin{array}{l}\text { Secondary } \\
\text { dendrite }\end{array}$ & $\begin{array}{l}\text { Tertiary } \\
\text { dendrite }\end{array}$ \\
\hline 9671.1 & & & 9 & 3 \\
\hline 9671.2 & 2 & 3 & & 2 \\
\hline 9671.3 & 3 & 4 & & \\
\hline 9671.4 & & 5 & & \\
\hline 9671.5 & & 3 & & \\
\hline 9671.6 & 3 & 6 & 2 & 5 \\
\hline 9671.7 & 2 & & & \\
\hline 9671.8 & 1 & 2 & & \\
\hline 9671.9 & 2 & & 7 & 2 \\
\hline 9671.10 & & & 1 & 2 \\
\hline 9672.1 & & & 4 & \\
\hline 9672.2 & & & 1 & \\
\hline 9672.3 & & 1 & & \\
\hline 9672.4 & 18 & 1 & 7 & \\
\hline 9672.5 & & & 4 & \\
\hline 9672.6 & & 3 & 2 & \\
\hline 9672.7 & & & 4 & \\
\hline 9672.8 & & & 6 & \\
\hline 9672.9 & 3 & & & \\
\hline 9672.10 & 9 & & & \\
\hline 9672.11 & 5 & 7 & 4 & 5 \\
\hline 9672.12 & & & 6 & \\
\hline 9672.13 & & & 3 & \\
\hline 9672.14 & 3 & 2 & 5 & 1 \\
\hline 9672.15 & & & 2 & \\
\hline 9672.16 & & 4 & & \\
\hline 9672.17 & & 1 & & \\
\hline
\end{tabular}

immunoreactive neurons were found to receive on average 6.7 boutons $(\mathrm{SD}=6.1)$ from an individual pallidal axon.

\section{Nitric oxide synthase-immunoreactive neurons}

A major target of some pallidostriatal axons were NOS-IR neurons (Table 3). In these cases, single axonal boutons or clusters of axonal boutons were often apposed to the soma and dendrites of individual NOS-IR neurons (Figs. 5C, $D, G, 6 A, H$, 7A; Table 3 ). Of 23 boutons examined by correlated light and electron microscopy, 21 were identified in synaptic contact with NOS-IR neurons (Figs. 6A,H, 7). In two cases the plane of section was not compatible with the verification of synaptic contact. Pallidal terminals were observed in symmetrical synaptic contact with the dendrites $(n=14)$ and soma $(n=7)$ of NOS-IR neurons (Figs. $6 A, H, 7)$. Individual terminals often possessed multiple synaptic release sites (Figs. 7E, $G$ ). Because the selectivity of pallidal axons for NOS-IR neurons was less consistent between animals (four of five GP neurons displayed varying degrees of selectivity for NOS-IR neurons) and less pronounced than for PV-IR neurons, a quantitative model of connectivity was not attempted.

\section{Postsynaptic targets of axon collaterals of pallidostriatal neurons in the substantia nigra}

The axon collateral of a pallidostriatal neuron in the substantia nigra (Fig. 1) was examined by correlated light and electron microscopy to determine whether a single pallidal neuron is also capable of forming synaptic contacts with dopaminergic (TH-IR) and GABAergic (PV-IR) neurons. Four neurons (three TH-IR neurons in the rostral pars compacta and one PV-IR neuron in the ventral-caudal pars reticulata) that were apposed by multiple pallidostriatal boutons were analyzed by correlated light and electron microscopy and were confirmed as receiving multiple symmetrical synaptic contacts to their proximal regions (Figs. 8, 9).

\section{DISCUSSION}

The results of the present study demonstrate that single GP neurons firing in a range of modes selectively innervate GABAergic and nitric oxide synthesizing interneurons of the neostriatum, and in addition they innervate diverse classes of neurons in the globus pallidus, entopeduncular nucleus, subthalamic nucleus, and substantia nigra. Thus single GP neurons have the potential to regulate the manner in which the basal ganglia receives, processes, and transmits cortical information.

\section{Firing patterns of juxtacellularly recorded pallidostriatal neurons}

Pallidostriatal neurons discharged at low or high rates and could fire in regular, irregular, and bursting modes. The faster firing neurons that discharge at rates greater than the maximal repetitive firing frequency of type $1 \mathrm{GP}$ neurons observed in guinea pig slices $(<10 \mathrm{~Hz})(\mathrm{Nambu}$ and Llinas, 1994) may correspond to type 2 neurons observed in the same preparation, which may fire repetitively up to $200 \mathrm{~Hz}$ with weak spike frequency adaptation (Nambu and Llinas, 1994). They may also correspond to the majority (73\%) of GP neurons recorded intracellularly in anesthetized rats that spontaneously fire repetitively $(2-40 \mathrm{~Hz})$, up to $60 \mathrm{~Hz}$ with steady-state current injection, and up to $500 \mathrm{~Hz}$ for short periods (Kita and Kitai, 1991). The ability of some of these neurons to fire bursts of action potentials (neurons 9645 and 9678) may relate to their expression of a low-threshold calcium conductance (Nambu and Llinas, 1994) and prevailing network activity in the anesthetized preparation (c.f. Steriade and Deschênes, 1984; Steriade, 1993).

Neurons firing at lower rates (neurons 9665 and 9671) may be composed of the type 1 neurons of Nambu and Llinas (1994) that discharge repetitively at $<10 \mathrm{~Hz}$ and can fire short bursts of rapidly accommodating spikes (mediated by a low-threshold calcium current and an A-current). However, we cannot exclude the possibility that they represent type 2 neurons (Nambu and Llinas, 1994), which appear to fire more slowly in the anesthetized preparation (Kita and Kitai, 1991; Ryan and Sanders, 1993) and may also discharge in bursts as described above. Taken together, these data suggest that the pallidostriatal neurons are composed of one or possibly two principal classes of neuron that may discharge in various patterns. This interpretation is consistent with the recent observation that both the type 1 and 2 neurons described by Nambu and Llinas (1997) projected to the neostriatum.

\section{General anatomical properties of pallidostriatal neurons}

The somatodendritic features of GP neurons that project to the neostriatum observed in this study are similar to those that have been described for the majority of neurons in the rat (Danner and Pfister, 1981; Falls and Park, 1981; Falls et al., 1983; Kita and Kitai, 1994) and guinea pig GP (Nambu and Llinas, 1997) and GPe of the primate (Fox et al., 1974; Park et al., 1982; François et al., 1984; Yelnik et al., 1984). Approximately onequarter of the GP neurons labeled in this study innervated the neostriatum, a figure that is comparable with other reports 

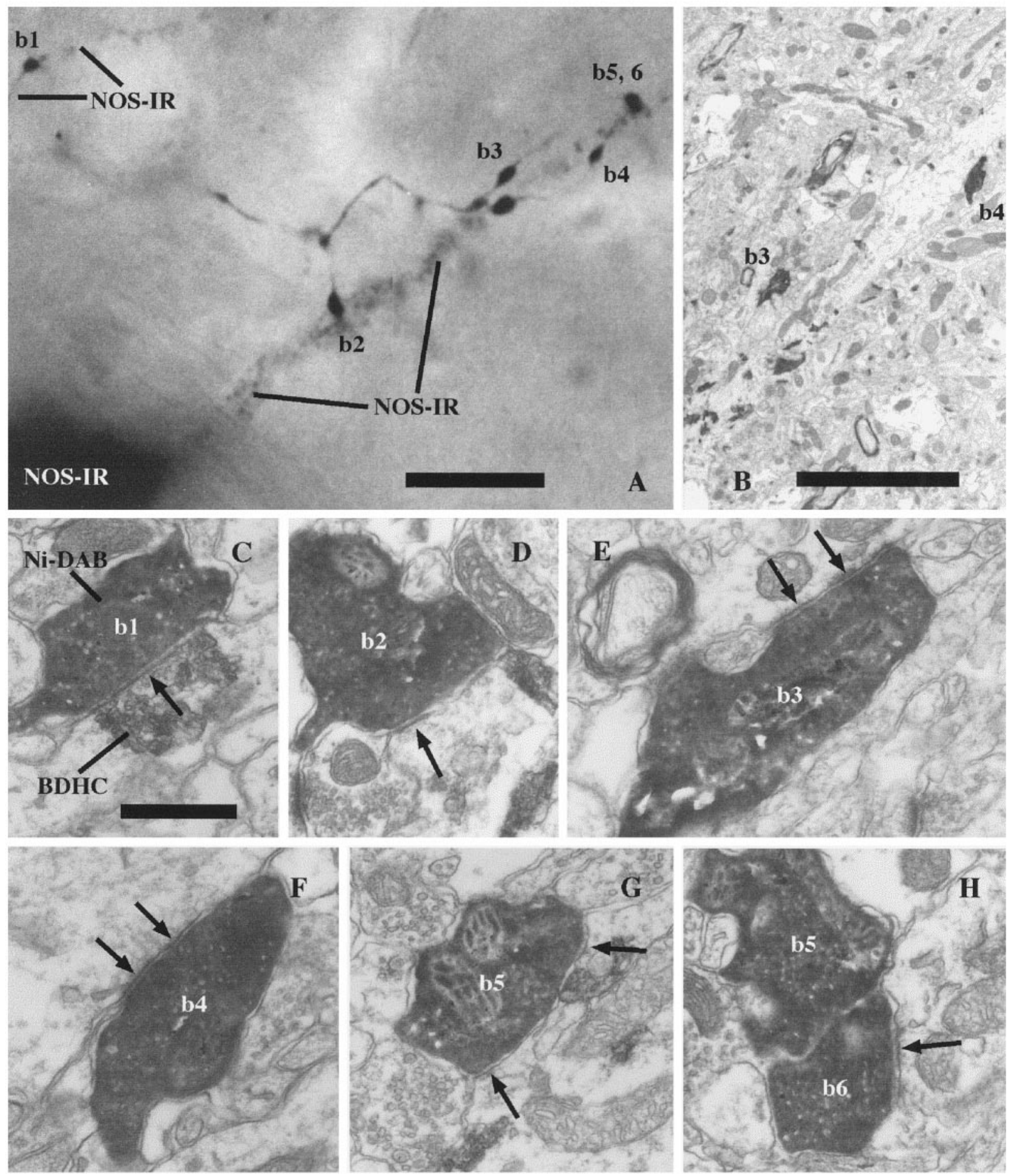

Figure 7. Light and electron micrographs illustrating the selective innervation of NOS-IR interneurons by an individual GP neuron 9678. $A$, Light microscopic montage of the dendrites of two NOS-IR interneurons (visualized with BDHC). One is apposed by one bouton (b1), and the other is apposed by five boutons $(b 2-b 6)$ of a densely labeled pallidostriatal axon visualized with Ni-DAB. $B$, Low-magnification electron micrograph illustrating part of the region in $A$. Note that $b 3$ and $b 4$ act as registration marks and $b 3$ apposes a branch of the dendrite to which $b 4$ is apposed. $C-H$, High-magnification electron micrographs of the boutons (b1-b6), illustrating their synaptic contacts with the two NOS-IR dendrites. Each bouton forms symmetrical synaptic contact with the NOS-IR dendrites identified by the BDHC reaction product (visible in $C, D$, and $G$ ). Note that individual boutons may possess multiple release sites $(E-G)$. The amorphous nature of the Ni-DAB product $(B-H)$ and the crystalline nature of the BDHC reaction product are apparent $(C$, $F, G)$. Scale bars: $A, 10 \mu \mathrm{m} ; B, 5 \mu \mathrm{m} ; C$ (also applies to $D-H), 0.5 \mu \mathrm{m}$. 


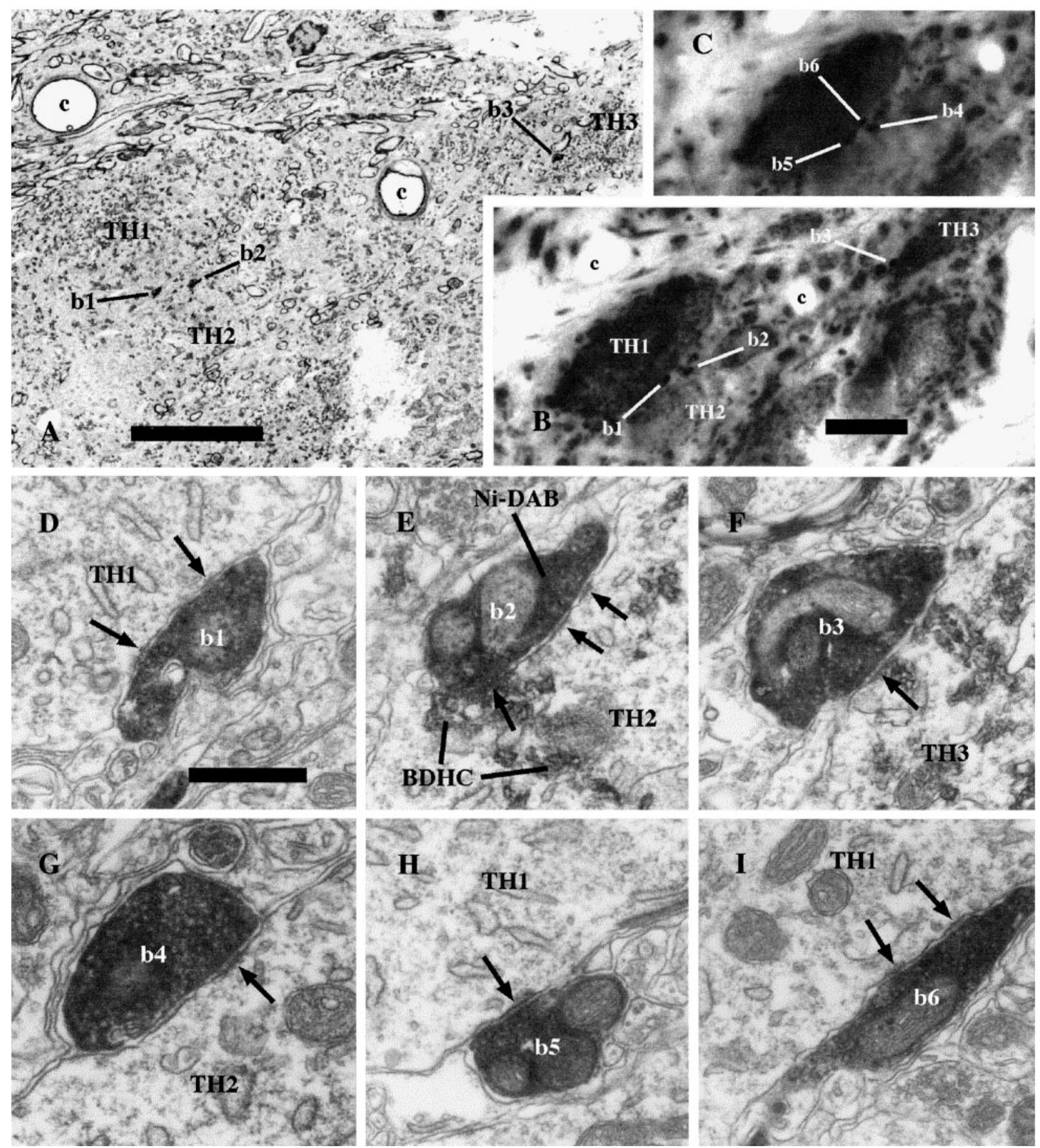

Figure 8. Light and electron micrographs illustrating the innervation of dopaminergic neurons of the substantia nigra pars compacta (TH1-TH3; visualized with BDHC) by pallidal neuron 9678 (visualized with Ni-DAB) that also innervated interneurons in the neostriatum (Figs. 6, 7) and also projected to the EP, STN, and SNr (Figs. 1, 6, 7, 9). A, Low-magnification electron micrograph of a region of the substantia nigra pars compacta that contains the somata of three TH-IR neurons ( $T H 2$ is lightly labeled) and three pallidonigral boutons (b1-b3). $B$, $C$, Light micrographs of the same region shown in $A$ at two different focal depths illustrating six boutons (b1-b6) apposed to the three TH-IR neurons. The two capillaries (c) act as further registration marks between the light and electron microscopes. $D-I$, High-magnification electron micrographs of the boutons (b1-b6) illustrating their synaptic contact with the three TH-IR neurons. Each bouton forms symmetrical synaptic contacts with the soma of the dopaminergic neurons (arrows). Note that individual boutons may possess multiple release sites $(D, E, I)$. In the electron micrographs the amorphous nature of the Ni-DAB product $(A$, $D-I)$ and the crystalline nature of the BDHC reaction product (visible in $E, F$ ) are apparent. Scale bars: $A, B, 10 \mu \mathrm{m} ; D($ also applies to $E-I$ ), $0.5 \mu \mathrm{m}$.

(Staines et al., 1981; Beckstead, 1983; Jayaraman, 1983; Staines and Fibiger, 1984; Smith and Parent, 1986; Walker et al., 1989; Kita and Kitai, 1994; Nambu and Llinas, 1997). This figure should be interpreted with caution, however, because (1) the recording and juxtacellular labeling method may select for subpopulations of neurons (Kita and Kitai, 1991; Ryan and Sanders, 1993; Pinault, 1996), (2) not all parts of the GP were sampled equally, and (3) the sample size is small. 

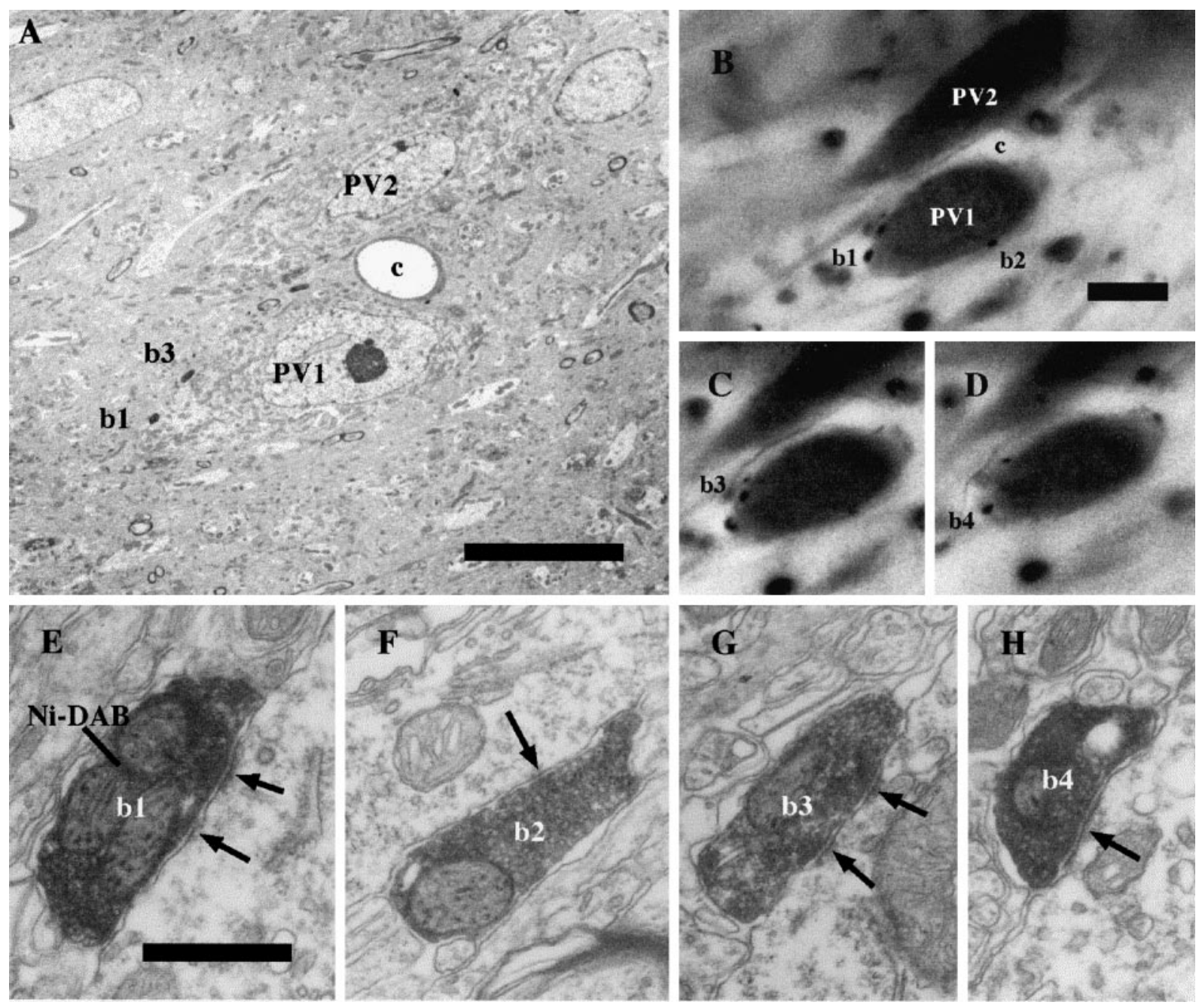

Figure 9. Light and electron micrographs illustrating the innervation of a PV-IR neuron of the substantia nigra pars reticulata by the axon terminals of pallidal neuron 9678 (visualized with NiDAB) that gave rise to the synaptic contacts in Figures 6, 7, and 8. $A$, Electron micrograph of a region of the substantia nigra pars reticulata that contains the somata of two PV-IR neurons ( $P V 1, P V 2$; visualized with DAB) and two boutons (b1, b3) that appose PV1. $B-D$, Light micrographs of the same region shown in $A$ at three different focal depths illustrating four boutons $(b 1-b 4)$ apposed to PV1. A capillary $(c)$ acts as a further registration mark of the light and electron micrographs. $E-H$, High-magnification electron micrographs of the boutons (b1-b4) illustrating their synaptic contacts with the soma of the PV-IR neuron PV1. Each bouton forms symmetrical synaptic contacts (arrows) with the soma of the GABAergic neuron. Note that individual boutons may possess multiple release sites $(E, G)$. In the electron micrographs $(A, E-H)$ the amorphous nature of the Ni-DAB reaction product is apparent. Scale bars: $A, B$ (also applies to $C, D$ ), $10 \mu \mathrm{m} ; D$ (also applies to $E-I$ ), $0.5 \mu \mathrm{m}$.

From the trajectory of single pallidostriatal axons that we observed and the results of retrograde and anterograde tracing in rats (Staines et al., 1981; Wilson and Phelan, 1982; Staines and Fibiger, 1984; Gerfen, 1985), cats (Beckstead, 1983; Jayaraman, 1983), and primates (Smith and Parent, 1986; Flaherty and Graybiel, 1993a), it is likely that the pallidostriatal projections and striatopallidal projections, which conserve dorsoventral and mediolateral relationships, are in register. Thus functionally related regions of GP and neostriatum are reciprocally connected.

Anterograde tracing studies from the GP/GPe have reported a projection to the neostriatum that has been described as sparse to substantial (Shu and Peterson, 1988; Kita et al., 1991; Rajakumar et al., 1994; Spooren et al., 1996). The reasons underlying these differences are not known but may be attributable in part to the high density of axons coursing through the GP and the possibility of the incorporation of tracers by fibers of passage and the possibility of multimodal transport (Smith et al., 1998). Through tracing of the labeled axon from the cell body of filled neurons, we confirm earlier reports (Kita and Kitai, 1994; Nambu and Llinas, 1997) that GP neurons do project to the neostriatum and their existence is not an artifact of extracellular tracing techniques.

We describe for the first time that the extent and density of pallidostriatal axons of individual neurons are highly heterogeneous, ranging from extensive and low-density arbors to more restricted and more dense focal arbors. Our data provide further evidence that an emergent property of individual pallidal neurons in subprimates (Staines and Fibiger, 1984; Kita and Kitai, 1994; Nambu and Llinas, 1997) and primates (Shink et al., 1996; Sato et al., 1997) is the arborization of their axon in multiple nuclei of the basal ganglia. Thus each GP neuron that projected to the neostriatum in this study arborized locally in the GP, and all five neurons that were traced caudally out of the GP also arborized in caudal basal ganglia targets. We have now demonstrated directly, through complete filling of single GP neurons in combination with correlated light and electron microscopy, that a single GP neuron that projects to the neostriatum may also form synaptic 
contacts with GABAergic output neurons of the substantia nigra pars reticulata and dopaminergic neurons of the substantia nigra pars reticulata, in addition to terminating in the GP, entopeduncular nucleus, and STN.

\section{Selectivity of pallidostriatal axons for neostriatal interneurons}

The most important finding of the present study is that each of the five pallidostriatal neurons that were studied selectively innervated interneurons of the neostriatum. Correlated light and electron microscopy confirmed that pallidal boutons apposing interneurons do indeed represent symmetrical synaptic contacts. They display ultrastructural features similar to those reported previously for pallidal terminals in other basal ganglia nuclei (for review, see Smith et al., 1998), i.e., they are of medium to large diameter, contain several mitochondria and variable densities of pleomorphic synaptic vesicles, and form symmetrical synaptic contacts with their postsynaptic targets, individual terminals often possessing more than one site of neurotransmitter release. Pallidal boutons innervated all parts of interneurons, characteristically forming clusters on restricted parts of the postsynaptic cell, suggesting that they powerfully influence membrane potential at specific points on the target cell, although the position and number of pallidal boutons on the population of interneurons innervated by a single axon were variable.

A large proportion of boutons (19-66\%) of an individual GP neuron in the neostriatum apposed PV-IR interneurons, despite the fact that these neurons represent only $\sim 3 \%$ of the total neuronal population in the neostriatum (Bolam et al., 1983, 1985; Cowan et al., 1990; Kita et al., 1990; Kita, 1991).

Selectivity of pallidostriatal axonal boutons for NOS-IR neurons [which are mostly equivalent to NADPH diaphorasereactive/somatostatin-IR interneurons (Kawaguchi et al., 1995; Kawaguchi, 1997) and represent $\sim 1 \%$ of the total striatal population (West et al., 1996)] has been reported previously on the basis of anterograde tracing from GP combined with NADPHdiaphorase histochemistry and biochemical studies (Staines and Hincke, 1991; Bennett et al., 1993). Our study confirms and extends this finding; four of five neurons displayed some degree of selectivity. The variable selectivity for NOS-IR neurons may be real or technical, the latter because of the variable quality of NOS immunolabeling obtained with our protocol.

The proportion of pallidostriatal boutons in contact with the two classes of neostriatal interneuron is likely to be an underestimate because immunostaining is usually less than $100 \%$ efficient because of the loss of antigenicity through processing and incomplete penetration of immunoreagents (Bolam, 1992). We attempted to adjust for the latter by only estimating the proportion of boutons in contact with interneurons at levels of the tissue where there was immunostaining. This approach may be biased, however, because of peroxidase reaction products, which diffuse more effectively in large diameter structures than smaller diameter structures or in tortuous structures.

Although each pallidostriatal neuron that was tested selectively innervated interneurons, it must be considered that because of the small sample size, the fact that not all regions of the GP were sampled, and the potential bias of our labeling technique, additional components of the pallidostriatal system may remain to be studied and these components may display different rules of connectivity. Furthermore, it remains to be determined whether components of the pallidostriatal projection of primates also display selectivity for interneurons.

\section{Functional implications}

Parvalbumin-immunoreactive interneurons represent the principal class of GABAergic interneuron in the neostriatum (Bolam et al., 1983, 1985; Cowan et al., 1990; Kita et al., 1990; Bennett and Bolam, 1994b). These interneurons mediate feed-forward inhibition (Kita, 1991; Pennartz and Kitai, 1991; Lapper et al., 1992; Bennett and Bolam, 1994a) and are involved in the synchronization of medium spiny projection neurons during periods of cortical activation (Plenz and Kitai, 1998). Because GP neurons receive monosynaptic and/or rapid disynaptic activation (via the STN) (Tremblay and Filion, 1989; Ryan and Clark, 1991; Kita, 1992; Naito and Kita, 1994; Plenz and Kitai, 1998) after cortical activation, they are well placed to modulate the cortical activation of PV interneurons (Pennartz and Kitai, 1991; Plenz and Kitai, 1998) through shunting of coincident cortical excitatory postsynaptic potentials and phase-lock action potential generation (c.f. Pennartz and Kitai, 1991; Cobb et al., 1995; Ulrich and Huguenard, 1997a,b). The total number and placement of GP terminals on PV interneurons when compared with studies of similar unitary inhibitory connections suggest that they might powerfully shunt excitatory inputs, phase-lock, or prevent action potential generation (Cobb et al., 1995). The same pallidostriatal neurons that innervate PV interneurons also provide major synaptic input to NOS interneurons, which themselves regulate neostriatal activity through the release of GABA (Kubota et al., 1993), nitric oxide (Hanbauer et al., 1992; Guevara-Guzman et al., 1994; Lonart and Johnson, 1994; East et al., 1996; Stewart et al., 1996), and neuropeptides (Radke et al., 1993), and to different classes of neurons in functionally related [and unrelated (Bevan et al., 1996, 1997a)] regions downstream of the neostriatum.

Given the topography of pallidostriatal axons and the variability in the placement and number of boutons on PV interneurons, it is likely that neighboring GP neurons that converge on a reciprocally connected region of the neostriatum must weight their connections to PV neurons appropriately so that the total pallidal input to an individual PV neuron is similar in number and placement. In addition, one might predict that presynaptic GP neurons fire synchronously to exert similar effects on PV neurons in a reciprocally connected zone of the neostriatum. However, there is little correlation of firing of neighboring GP neurons in awake behaving animals (Nini et al., 1995), suggesting that the pallidostriatal projection may equally exert complex, heterogeneous effects on interneurons in the same functional region of the neostriatum. The diversity of pallidostriatal axonal arborizations in terms of their extent and numbers of boutons may relate to the complex patch-striosome/matrix (Graybiel, 1990; Gerfen, 1992) and matrix/matrisome compartmentation (Flaherty and Graybiel, 1991, 1993a,b, 1994, 1995) of the neostriatum. Future studies combining filling of single GP neurons in combination with staining of markers of neostriatal compartmentation are required to test this hypothesis. The nature of the synaptic targets of pallidostriatal boutons that innervated immunonegative structures also remains to be established. Do these targets represent medium spiny projection neurons (Kita et al., 1991; Rajakumar et al., 1994), other classes of interneuron, or PV and NOS interneurons that were not labeled for technical reasons?

In conclusion, the findings we present, taken together with previous data, suggest that GP neurons play an important role in synchronizing and spatially and temporally structuring the complex and dynamic response of the whole basal ganglia in response to cortical activation during behavior. 


\section{REFERENCES}

Albin RL, Young AB, Penny JB (1989) The functional anatomy of basal ganglia disorders. Trends Neurosci 12:366-375.

Anderson ME, Turner RS (1991) A quantitative analysis of pallidal discharge during targeted reaching movement in the monkey. Exp Brain Res 86:623-632.

Beckstead RM (1983) A pallidostriatal projection in the cat and monkey. Brain Res Bull 11:629-632.

Bennett BD, Bolam JP (1994a) Synaptic input and output of parvalbumin-immunoreactive neurones in the neostriatum of the rat. Neuroscience 62:707-719.

Bennett BD, Bolam JP (1994b) Localisation of parvalbuminimmunoreactive structures in primate caudate-putamen. J Comp Neurol 347:340-356.

Bennett BD, Bacon S, Bolam JP (1993) Identified targets of the pallidostriatal projection in the rat. Soc Neurosci Abstr 19:1432.

Bevan MD, Bolam JP, Crossman AR (1994a) Convergent synaptic input from the neostriatum and the subthalamus on to identified nigrothalamic neurons in the rat. Eur J Neurosci 6:320-334.

Bevan MD, Crossman AR, Bolam JP (1994b) Neurons projecting from the entopeduncular nucleus to the thalamus receive convergent synaptic inputs from the subthalamic nucleus and the neostriatum. Brain Res 659:99-109.

Bevan MD, Smith AD, Bolam JP (1996) The substantia nigra as a site of synaptic integration of functionally diverse information arising from the ventral pallidum and the globus pallidus in the rat. Neuroscience 75:5-12.

Bevan MD, Clarke NP, Bolam JP (1997a) Synaptic integration of functionally diverse pallidal information in the entopeduncular nucleus and subthalamic nucleus in the rat. J Neurosci 17:308-324.

Bevan MD, Eaton SAE, Bolam JP (1997b) Synaptic targets of physiologically, neurochemically and morphologically characterized neurons of the rat globus pallidus. Soc Neurosci Abstr 23:196.

Bolam JP (1992) Experimental neuroanatomy. Oxford: Oxford UP.

Bolam JP, Smith Y (1992) The striatum and the globus pallidus send convergent synaptic inputs onto single cells in the entopeduncular nucleus of the rat: a double anterograde labelling study combined with post-embedding immunocytochemistry for GABA. J Comp Neurol 321:456-476.

Bolam JP, Clarke DJ, Smith AD, Somogyi P (1983) A type of aspiny neuron in the rat neostriatum accumulates $\left({ }^{3} \mathrm{H}\right) \gamma$-aminobutyric acid: combination of Golgi-staining, autoradiography and electron microscopy. J Comp Neurol 213:121-134.

Bolam JP, Powell JP, Wu JY, Smith AD (1985) Glutamate decarboxylase-immunoreactive structures in the rat neostriatum. A correlated light and electron microscopic study including a combination of Golgi-impregnation with immunocytochemistry. J Comp Neurol 237:1-20.

Chevalier G, Deniau JM (1990) Disinhibition as a basic process in the expression of striatal functions. Trends Neurosci 13:277.

Chockkan V, Mewes K, Zhang JY, Starr P, Bakay RAE, DeLong M, Vitek JL (1997) A comparison of discharge pattern of internal segment neurons of the globus pallidus (GPi) in akinetic and tremor parkinsonian patients (PD). Soc Neurosci Abstr 23:470.

Cobb SR, Buhl EH, Halasy K, Paulsen O, Somogyi P (1995) Phasing of neuronal activity by individual hippocampal GABAergic interneurons. Nature 378:75-78.

Cowan RL, Wilson CJ, Emson PC, Heizmann CW (1990) Parvalbumincontaining GABAergic interneurons in the rat neostriatum. J Comp Neurol 302:197-205.

Crossman AR (1989) Neural mechanisms in disorders of movement. Comp Biochem Physiol 93A:141-149.

Danner H, Pfister C (1981) Untersuchungen zur zytoarchitektonik des globus pallidus der ratte. J Hirnforsch 22:47-57.

DeLong MR (1971) Activity of pallidal neurons during movement. J Neurophysiol 34:414-427.

DeLong MR (1990) Primate models of movement disorders of basal ganglia origin. Trends Neurosci 13:281-285.

DeLong MR, Alexander GE, Mitchell SJ, Richardson RT (1986) The contribution of basal ganglia to limb control. Prog Brain Res 64:161-174.

East SJ, Parry-Jones A, Brotchie JM (1996) Ionotropic glutamate receptors and nitric oxide synthesis in the rat striatum. NeuroReport 8:71-75.

Falls WM, Park MR (1981) Light and EM analysis of rat globus pallidus neurons intracellularly recorded and labelled with HRP. Anat Rec 199:79-80.

Falls W M, Park MR, Kitai ST (1983) An intracellular HRP study of the rat globus pallidus. II. Fine structural characteristics and synaptic connections of medially located large globus pallidus neurons. J Comp Neurol 221:229-245.

Filion M (1979) Effects of interruption of the nigrostriatal pathway and of dopaminergic agents on the spontaneous activity of globus pallidus neurons in the awake monkey. Brain Res 178:425-441.

Flaherty AW, Graybiel AM (1991) Corticostriatal transformations in the primate somatosensory system. Projections from physiologically mapped body-part representations. J Neurophysiol 66:1249-1263.

Flaherty AW, Graybiel AM (1993a) Output architecture of the primate putamen. J Neurosci 13:3222-3237.

Flaherty AW, Graybiel AM (1993b) Two input systems for body representations in the primate striatal matrix: experimental evidence in the squirrel monkey. J Neurosci 13:1120-1137.

Flaherty AW, Graybiel AM (1994) Input-output organization of the sensorimotor striatum in the squirrel monkey. J Neurosci 14:599-610.

Flaherty AW, Graybiel AM (1995) Motor and somatosensory corticostriatal projection magnifications in the squirrel monkey. J Neurophysiol 74:2638-2648.

Fox CA, Andrade AN, Luiqui IJ, Rafols JA (1974) The primate globus pallidus: a Golgi and electron microscopic study. J Hirnforsch 15:75-93.

François C, Percheron G, Yelnik J, Heyner S (1984) A Golgi analysis of the primate globus pallidus. I. Inconstant processes of large neurons, other neuronal types, and afferent axons. J Comp Neurol 227:182-199.

Fujimoto K, Kita H (1992) Responses of rat substantia nigra pars reticulata units to cortical stimulation. Neurosci Lett 142:105-109.

Fujimoto K, Kita H (1993) Response characteristics of subthalamic neurons to stimulation of the sensorimotor cortex in the rat. Brain Res 609:189-192.

Gerfen CR (1985) The neostriatal mosaic. I. Compartmental organization of projections from the striatum to the substantia nigra in the rat. J Comp Neurol 236:454-476.

Gerfen CR (1992) The neostriatal mosaic: multiple levels of compartmental organization in the basal ganglia. Annu Rev Neurosci 15:285-320

Gerfen CR, Wilson CJ (1996) The basal ganglia. In: Integrated systems of the CNS, Part III. Handbook of chemical neuroanatomy 12 (Björklund A, Swanson L, Hökfelt T, eds), pp 369-466. Amsterdam: Elsevier Science.

Graybiel AM (1990) Neurotransmitters and neuromodulators in the basal ganglia. Trends Neurosci 13:244-254.

Guevara-Guzman R, Emson PC, Kendrick KM (1994) Modulation of in vivo striatal transmitter release by nitric oxide and cyclic GMP. J Neurochem 62:807-810.

Hanbauer I, Wink D, Osawa Y, Edelman GM, Gally JA (1992) Role of nitric oxide in NMDA-evoked release of ${ }^{3} \mathrm{H}$-dopamine from striatal slices. NeuroReport 3:409-412.

Herbison AE, Simonian SX, Norris PJ, Emson PC (1996) Relationship of neuronal nitric oxide synthase immunoreactivity to GnRH neurons in the ovariectomized and intact female rat. $\mathrm{J}$ Neuroendocrinol $8: 73-82$.

Jayaraman A (1983) Topographic organization and morphology of peripallidal and pallidal cells projecting to the striatum in cats. Brain Res 275:279-286.

Johnson DH (1996) Point process models of single-neuron discharge. J Comp Neurosci 3:275-299.

Kaneoke Y, Vitek JL (1996) Burst and oscillation as disparate neuronal properties. J Neurosci Methods 68:211-223.

Kawaguchi Y (1997) Neostriatal cell subtypes and their functional roles. Neurosci Res 27:1-8.

Kawaguchi Y, Wilson CJ, Augood SJ, Emson PC (1995) Striatal interneurones: chemical, physiological and morphological characterization. Trends Neurosci 18:527-535.

Kita H (1991) GABAergic circuits of the neostriatum. In: Chemical signalling in the basal ganglia, progress in brain research 99 (Arbuthnott GW, Emson PC, eds), pp 51-72. Amsterdam: Elsevier.

Kita H (1992) Responses of globus pallidus neurons to cortical stimulation: intracellular study in the rat. Brain Res 589:84-90.

Kita H, Kitai ST (1991) Intracellular study of rat globus pallidus neurons: membrane properties and responses to neostriatal, subthalamic and nigral stimulation. Brain Res 564:296-305.

Kita H, Kitai ST (1994) The morphology of globus pallidus projection 
neurons in the rat: an intracellular staining study. Brain Res 636:308-319.

Kita H, Kosaka T, Heizmann CW (1990) Parvalbumin-immunoreactive neurons in the rat neostriatum: a light and electron microscopic study. Brain Res 536:1-15.

Kita H, Chang HT, Fujimoto K (1991) Pallidal-neostriatal projections of the rat. Soc Neurosci Abstr 17:453.

Kubota Y, Mikawa S, Kawaguchi Y (1993) Neostriatal GABAergic interneurones contain NOS, calretinin or parvalbumin. NeuroReport 5:205-208.

Lapper SR, Smith Y, Sadikot AF, Parent A, Bolam JP (1992) Cortical input to parvalbumin-immunoreactive neurones in the putamen of the squirrel monkey. Brain Res 580:215-224.

Lonart G, Johnson KM (1994) Inhibitory effects of nitric oxide on the uptake of ${ }^{3} \mathrm{H}$-dopamine and ${ }^{3} \mathrm{H}$-glutamate by striatal synaptosomes. J Neurochem 63:2108-2117.

Naito A, Kita H (1994) The cortico-pallidal projection in the rat: an anterograde tracing study with biotinylated dextran amine. Brain Res 653:251-257.

Nambu A, Llinas R (1994) Electrophysiology of globus pallidus neurons in vitro. J Neurophysiol 72:1127-1139.

Nambu A, Llinas R (1997) Morphology of globus pallidus neurons: its correlation with electrophysiology in guinea pig brain slices. J Comp Neurol 377:85-94.

Nini A, Feingold A, Slovin H, Bergman H (1995) Neurons in the globus pallidus do not show correlated activity in the normal monkey, but phase-locked oscillations appear in the MPTP model of Parkinsonism. J Neurophysiol 74:1800-1805.

Park MR, Falls WM, Kitai ST (1982) An intracellular HRP study of the rat globus pallidus. I. Responses and light microscopic analysis. J Comp Neurol 211:284-294.

Pennartz CMA, Kitai ST (1991) Hippocampal inputs to identified neurons in an in vitro slice preparation of the rat nucleus accumbens: evidence for feed-forward inhibition. J Neurosci 11:2838-2847.

Pinault D (1996) A novel single-cell staining procedure performed in vivo under electrophysiological control: morpho-functional features of juxtacellularly labelled thalamic cells and other central neurons with biocytin or neurobiotin. J Neurosci Methods 65:113-136.

Plenz D, Kitai ST (1998) Up and down states in striatal medium spiny neurons simultaneously recorded with spontaneous activity in fastspiking interneurons studied in cortex-striatum-substantia nigra organotypic cultures. J Neurosci 18:266-283.

Radke JM, Spyraki C, Thermos K (1993) Neuronal release of somatostatin in the rat striatum: an in vivo microdialysis study. Neuroscience 54:493-498.

Rajakumar N, Elisevich K, Flumerfelt BA (1994) The pallidostriatal projection in the rat: a recurrent inhibitory loop? Brain Res 651:332-336.

Ryan LJ, Clark KB (1991) The role of the subthalamic nucleus in the response of globus pallidus neurons to stimulation of the prelimbic and agranular frontal cortices in rats. Exp Brain Res 86:641-651.

Ryan LJ, Clark KB (1992) Alteration of neuronal responses in the subthalamic nucleus following globus pallidus and neostriatal lesions in rat. Brain Res Bull 29:319-327.

Ryan LJ, Sanders DJ (1993) Subthalamic nucleus lesion regularizes firing patterns in globus pallidus and substantia nigra pars reticulata neurons in rats. Brain Res 626:327-331.

Ryan LJ, Sanders DJ (1994) Subthalamic nucleus and globus pallidus lesions alter activity in nigrothalamic neurons in rats. Brain Res Bull 34:19-26.

Sato F, Levesque M, Nakamura Y, Parent A (1997) Axonal projections of single cells from the external pallidum in monkeys. Soc Neurosci Abstr 23:196.

Shink E, Bevan MD, Bolam JP, Smith Y (1996) The subthalamic nucleus and the external segment of the globus pallidus: two tightly interconnected structures that control the output of the basal ganglia in the monkey. Neuroscience 73:335-357.

Shu SY, Peterson GM (1988) Anterograde and retrograde axonal transport of Phaseolus vulgaris-leucoagglutinin (PHA-L) from the globus pallidus to the striatum of the rat. J Neurosci Methods 25:175-180.

Smith Y, Bolam JP (1991) Convergence of synaptic inputs from the striatum and the globus pallidus onto identified nigrocollicular cells in the rat: a double anterograde labelling study. Neuroscience 44:45-73.

Smith Y, Parent A (1986) Differential connections of caudate nucleus and putamen in the squirrel monkey (Saimiri sciureus). Neuroscience 18:347-371.

Smith Y, Wichmann T, DeLong MR (1994) Synaptic innervation of neurones in the internal pallidal segment by the subthalamic nucleus and the external pallidum in monkeys. J Comp Neurol 343:297-318.

Smith Y, Bevan MD, Shink E, Bolam JP (1998) Microcircuitry of the direct and indirect pathways of the basal ganglia. Neuroscience 86:353-387.

Spooren WPJM, Lynd-Balta E, Mitchell S, Haber SN (1996) Ventral pallidostriatal pathway in the monkey; evidence for modulation of basal ganglia circuits. J Comp Neurol 370:295-312.

Staines WA, Fibiger HC (1984) Collateral projections of neurons of the rat globus pallidus to the striatum and substantia nigra. Exp Brain Res 56:217-220.

Staines WA, Hincke MTC (1991) Substantial alterations in neurochemical and metabolic indices in select basal ganglia neurons following lesions of globus pallidus neurons in rats. Soc Neurosci Abstr 17:453.

Staines WA, Atmadja S, Fibiger HC (1981) Demonstration of a pallidostriatal pathway by retrograde transport of HRP-labelled lectin. Brain Res 206:446-450.

Steriade M (1993) Cellular substrates of brain rhythms. In: Electroencephalography. Basic principles, clinical applications, and related fields (Niedermeyer E, Lopes Da Silva F, eds), pp 27-62. Baltimore: Williams \& Wilkins.

Steriade M, Deschênes M (1984) The thalamus as a neuronal oscillator. Brain Res Rev 8:1-63.

Stewart TL, Michel AD, Black MD, Humphrey PPA (1996) Evidence that nitric oxide causes calcium-independent release of ${ }^{3} \mathrm{H}$-dopamine from rat striatum in vitro. J Neurochem 66:131-137.

Tremblay L, Filion M (1989) Responses of pallidal neurons to striatal stimulation in intact waking monkeys. Brain Res 498:1-16.

Turner RS, Anderson ME (1997) Pallidal discharge related to the kinematics of reaching movements in two dimensions. J Neurophysiol 77:1051-1074.

Ulrich D, Huguenard JR (1997a) GABA-A receptor mediated rebound burst firing and shunting in thalamus. J Neurophysiol 78:1748-1751.

Ulrich D, Huguenard JR (1997b) Nucleus specific chloride homeostasis in rat thalamus. J Neurosci 17:2348-2354.

Walker RH, Arbuthnott GW, Wright AK (1989) Electrophysiological and anatomical observations concerning the pallidostriatal pathway in the rat. Exp Brain Res 74:303-310.

West MJ, Ostergaard K, Andreasssen OA, Finsen B (1996) Estimation of the number of somatostatin neurons in the striatum: an in situ hybridization study using the fractionator method. J Comp Neurol 370:11-22.

Whittington MA, Traub RD, Jefferys JGR (1995) Synchronized oscillations in interneuron networks driven by metabotropic glutamate receptor activation. Nature 373:612-615.

Wilson CJ, Phelan KD (1982) Dual topographic representation of neostriatum in the globus pallidus of rats. Brain Res 243:354-359.

Yelnik J, Percheron G, François C (1984) A Golgi analysis of the primate globus pallidus. II. Quantitative morphology and spatial orientation of dendritic arborizations. J Comp Neurol 227:200-213. 